Pensamiento Crítico Vol. 18 N 2, pp. 77-94

\title{
Efectos de la política monetaria de flexibilización cuantitativa en Estados Unidos de América sobre el empleo
}

\author{
Carlos Palomino Selem
}

\section{RESUMEN}

El Sistema de la Reserva Federal de los Estados Unidos de América (Fed) con el propósito de hacer posible la recuperación económica y colocar ha EE.UU. en la senda del crecimiento económico sostenido, desarrollo los programas de flexibilidad cuantitativa (quantitative easing -QE-) los mismo que tuvieron por objetivo reducir la tasa de desempleo a 6.5\% para el 2014; esto se podría conseguir si el Fed desarrollaba un programa de compras de activos, lo que significa ingresar dinero de primer poder a la economía, levantando el balance del Fed. Planteado de esta forma el Fed anclaba la política monetaria a la evolución del empleo, expresado en la tasa de desempleo.

Los resultados no han sido decepcionantes, todo lo contrario, pero sobre los objetivos del empleo no se ha llegado a finales del 2013 a una cifra cercana a la planteada de $6.5 \%$ y el último resultado se ubicó en $7 \%$ de tasa de desempleo; aún así el Fed en su reunión de diciembre del 2013 empezó el recorte del programa de estímulos monetarios.

El presente documento, examina la relación que ha tomado el QE con el empleo; particularmente con la creación de empleo no agrícola (Non Farm Payroll -NFP-). Los resultados que hemos obtenido muestran un grado de relación importante 


\section{Pensamiento Crítico Vol. 18. No 2}

pero no contundente. Consideramos que la administración de la política monetaria no ha sido en todo momento muy consistente con la situación de coyuntura con que los eventos en los mercados se presentaban y desenvolvía ${ }^{\circ}$

Palabras claves: Comité federal de mercados abiertos, política monetaria, reducción gradual, política acomodaticia, flexibilización cuantitativa, política acomodaticia; reducción gradual, flexibilización cuantitativa, sistema de la reserva federal, el balance de la FED, operaciones de mercado abierto, momento óptimo timing, creación de nuevos puestos de trabajo no agrícola, prueba de normalidad.

\section{ABSTRACT}

The Federal Reserve System has the purpose to get the recovery economic and putting to the EE.UU.in the path to sustainable economic growth, for this the Fed come developing the quantitative easing programs, the same have for objective reduce unemployment rate to $6.5 \%$ for 2014 ; this could get, if the Fed designs a purchase program of assets; this mean inject of first power money to the economy, raising the balance of the Fed. In this way the Fed drops anchor the monetary policy to the evolution of the employ, this is expressed in the unemployment rate.

The results hasn't been disappointing, but the employment objectives did not reach to the goals of the 2013 around of $6.5 \%$, the last result was 7.\%. Still the Fed in its december 2013 meeting began the tapering of monetary stimulus programs.

This article, we study the relationship between Qe and the employ; specially with the Non Farm Payroll. The results we have obtained show us some grade of important relationship but it isn't overwhelming. We consider that the management of monetary policy has not been very consistent all the time with the conjuncture needs that market events would be unwrapped.

Keywords: Federal open market committing, monetary policy, tapering, accommodative policy, quantitative easing, federal reserve system, the balance of fed, open market operations, timing, nonfarm payroll; normally test.

\section{INTRODUCCIÓN}

La presente investigación plantea el problema de la efectividad de la política monetaria para generar o crear empleo. Este es un tema que el Comité Federal de Mercados Abiertos (FOMC por sus siglas en Inglés). En diversos pronunciamientos de la 


\section{Carlos Palomino Selem}

presentación del Presidente del FOMC el Sr. Ben Bernanke (ver FOMC Meetings 2013) expresó el objetivo de alcanzar el 6.5\% de tasa de desempleo en Estados Unidos.

En este sentido el informe técnico presentado aquí incorpora nuestra hipótesis de trabajo que se refiere a la relación entre la política monetaria expansiva del FOMC, que se considera como programa de estímulos monetarios por su carácter acomodaticio; es decir, de apoyo a el desempeño de la actividad económica y el empleo, de manera que sea un soporte a salir de la recesión y mantener el proceso de recuperación hasta que se consolide la recuperación económica y se inicie un proceso de crecimiento económico, el mismo que llamaría un periodo de retiro gradual de los estímulos monetarios (Cox 2013), denominado tapering en Íngles.

En los encuentros realizados por el FOMC en los denominados press release del FOMC Meetings (2013) se discutió sobre el problema de los objetivos de empleo y política monetaria.

Para probar nuestra hipótesis hemos formulado tres modelos, los mismos que no han mostrado resultados con significancia estadística. Se ha agregado un análisis de eventos que facilitan interpretar el motivo por el cual los modelos no han permitido explicar consistentemente el desempeño de las variables estudiadas.

\section{PLANTEAMIENTO DEL ESTUDIO}

La presente investigación tiene por objeto analizar la política de monetaria de flexibilización cuantitativa (Quantitative Easing), diseñada e implementada por el Sistema de la Reserva Federal (Fed. el banco central de los EE.UU. y su impacto sobre el empleo (estimado por la Creación de Nuevos Puestos de Trabajo No Agrícola, NFP por sus siglas en Inglés) y la producción expresada por el Producto Bruto Interno (PBI) -Chandler (2013)-, para los Estados Unidos de América (EE.UU.).

La importancia de la investigación radica en que entre los objetivos de política económica de la Fed, la meta que se alcance en el empleo permitirá dar fortaleza a la demanda agregada y al desempeño del nivel de actividad económica. 


\section{Pensamiento Crítico Vol. 18. No 2}

Para la Fed, el objetivo es que se consiga una tasa de desempleo de $6.5 \%$, nivel que indicaría que la recuperación económica en EE.UU. se habría consolidado; es decir, la tasa de desempleo se ha convertido en un indicador de anclaje para la economía.

\section{MARCO TEÓRICO}

Se considera que la Fed interviene en el nivel de actividad económica a través de la financiación del sector gobierno y sector privado. En este sentido, la Fed mediante operaciones de mercado abierto afecta la base monetaria o Balance del Fed. (B) de la forma siguiente:

$$
\mathrm{EP}=\mathrm{B}
$$

Siendo EP la emisión primaria donde:

$$
\mathrm{EP}=\mathrm{RIN}+\mathrm{CI}+\mathrm{OM}
$$

Las reservas internacionales netas (incluye el oro monetario) es "RIN"; el crédito interno neto"CIN", el cual son los préstamos que realiza la Fed. hacia la banca privada; y OM corresponde a las operaciones de mercado abierto, compra o venta de instrumentos financieros por parte del banco central al sector privado, publico y lo que se denomina resto del mundo.

Además:

$$
\Delta \mathrm{B}_{\mathrm{t}}=\Delta \mathrm{B}(\mathrm{T})_{\mathrm{t}}+\Delta \mathrm{B}(\mathrm{F})_{\mathrm{t}}
$$

Siendo $\Delta \mathrm{B}_{\mathrm{t}}$ el incremento de la base monetaria o balance del banco central en el momento " $\mathrm{t}$ " colocada una parte, para fines de nuestro estudio, en $\Delta \mathrm{B}(\mathrm{T})_{\mathrm{t}}$, incremento de la base monetaria debido a compras de la Fed. de instrumentos del Tesoro Público de los EE.UU. en el momento t; y la otra parte, asignada a $\Delta \mathrm{B}(\mathrm{F})_{\mathrm{t}}$ incremento de la $\mathrm{B}$ por compras de instrumentos del sector privado financiero.

Este incremento en $\Delta \mathrm{B}_{\mathrm{t}}$ tendrá impacto en el nivel de actividad económica expresado en el Producto bruto interno PBI mediante la relación: 


\section{Carlos Palomino Selem}

$$
\Delta \mathrm{PBI}_{\mathrm{t}}=\varphi\left[\Delta \mathrm{M}_{\mathrm{t}-\mathrm{k}}\right] *\left[1 / \mathrm{P}_{\mathrm{t}}\right]
$$

Donde $\varphi$ es una constante, $\Delta \mathrm{M}_{\mathrm{t}-\mathrm{k}}$ resulta en el incremento de la oferta monetaria con un rezago de k periodos, y $\mathrm{P}$ es el índice de precios; el $\Delta \mathrm{M}_{\mathrm{t}-\mathrm{k}}$ se debe a que:

$$
\Delta \mathrm{M}_{\mathrm{t}-\mathrm{k}}=\mathrm{m} * \Delta \mathrm{B}_{\mathrm{t}-\mathrm{k}}
$$

El efecto sobre el empleo tendría como mecanismo de transmisión la expresión de Okun:

$$
\begin{aligned}
& \Delta \mu_{t}=-\theta *\left[\mathrm{PBI}_{\mathrm{t}}-\mathrm{PBI}_{\mathrm{t}-\mathrm{k}}\right] \\
& \Delta \mu_{\mathrm{t}}=-\theta * \Delta \mathrm{PBI}_{\mathrm{t}}
\end{aligned}
$$

Siendo $\Delta \mu_{\mathrm{t}}$ la variación en la tasa de desempleo y $\theta$ una constante.

Reemplazando (6 $6^{a}$ y (5) en (4) se obtiene:

$$
\Delta \mu_{\mathrm{t}}=-\theta \varphi *\left[m * \frac{B_{t-k}}{P_{t}}\right]
$$

\section{HIPÓTESIS}

Nuestra hipótesis se construyó en base a un modelo que buscó relacionar que la política monetaria de flexibilización cuantitativa $(\mathrm{QE})$, definida por un programa importante, en su monto, de compras de bonos al sector público y privado financiero, tuvo efectos limitados o no consistentes en el tiempo sobre el incremento del empleo y el PBI.

\section{METODOLOGÍA Y TÉCNICAS DE INVESTIGACIÓN UTILIZADAS}

Hemos trabajado un conjunto de modelos de regresión con rezagos de un mes y un trimestre $(\mathrm{k}=1)$ dependiendo de las variables consideradas: NFP y PBI respectivamente; esto bajo las modalidades de regresiones con primeras y segundas variaciones porcentuales. 


\section{Pensamiento Crítico Vol. 18. No 2}

Los tamaños muestrales han variado, aplicándose a periodos desde 2003-Q4 hasta el 2013-Q2 con observaciones trimestrales para análisis en que intervenga el PBI; esto para confirmar comportamientos estructurales; y para periodos muestrales más cortos desde el 2008-Enero hasta 2013-Mayo para análisis de tipo condicional dado la aplicación del QE, con la finalidad de evaluar la política monetaria de QE diseñada e implementada por la Fed. para influir sobre el empleo.

Los periodos de QE son los siguientes: El QE-1 abarca de Enero 2009 a marzo el 2010; El QE-2 comprende desde noviembre del 2010 hasta junio del 2011; y El QE-3 que corresponde desde septiembre 2012 hasta la actualidad.

Las variables sujetas a análisis son las siguientes:

1. La Balance del Fed. (B).

2. El Producto Bruto Interno (PBI).

3. La Creación de Puestos de Trabajo No Agrícolas (Non Farm Payrol-NFP-).

\section{RECOLECCIÓN E INTERPRETACIÓN DE DATOS}

\section{Hechos Estilizados (Style Fact)}

Para el periodo de estudio se tomaron los años que van entre el 2008-Enero y el 2013-Mayo; tiempo que comprende la implementación de la política de flexibilización cuantitativa (QE) con la finalidad de salir de la recesión en EE.UU. impulsando el empleo con la finalidad de alcanzar la consolidación del crecimiento del nivel de actividad económica.

El crecimiento significativo de B (ver GRÁFICO N 1 ) como consecuencia del QE-1 fue notable; rol similar cumplió el QE-2; e igualmente lo viene haciendo el QE-3; pero los resultados obtenidos sobre el empleo obtenidos en el periodo de análisis, fueron menores a lo esperado. 


\section{Carlos Palomino Selem}

GRÁFICO N 1 . Balance de la Reserva Federal por periodos de QE (Billones de US\$)

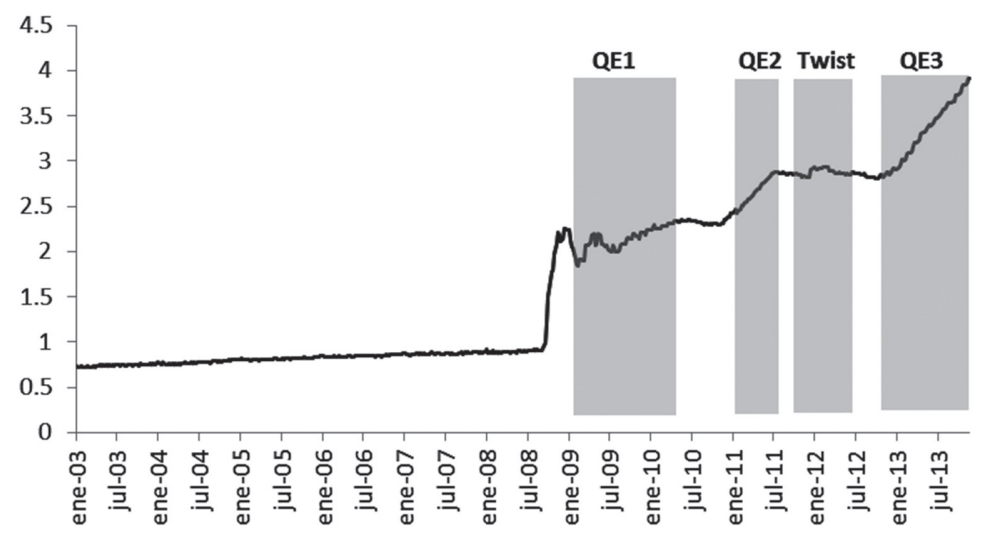

Fuente: Federal Reserve

Elaboración propia.

Lo que, también, se puede distinguir es que el NFP vino incrementándose en la mayor parte del proceso del QE (ver GRÁFICO № 2); aunque para la Fed. no se lograron alcanzar los objetivos de empleo propuestos por el Comité Federal de Mercados Abiertos (FOMC).

GRÁFICO Nº 2. Nom Farm Payrolls

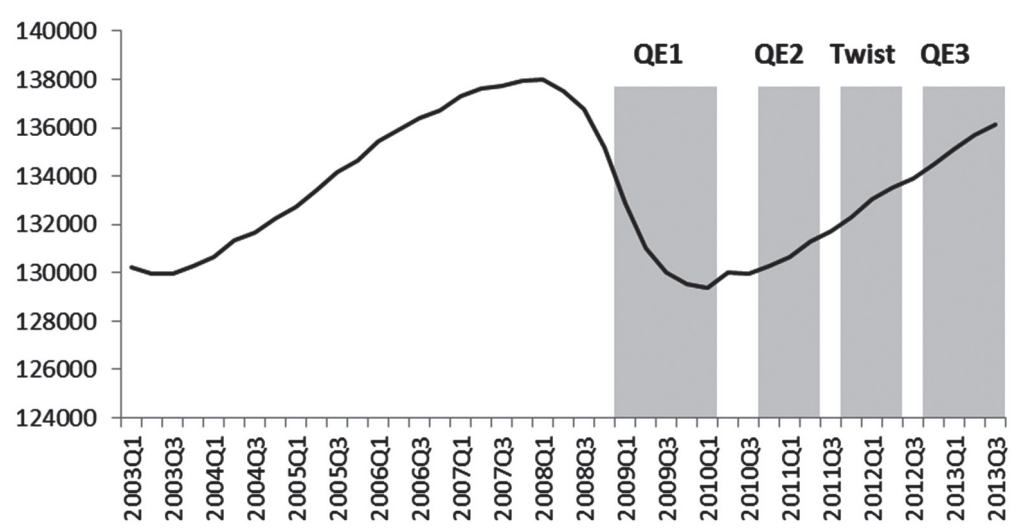

Fuente: Federal Reserve y US Bureau of Labor Statistic

Elaboración propia. 


\section{Pensamiento Crítico Vol. 18. No 2}

La correlación entre el NFP y el B durante el QE (ver GRÁFICO № 3) es bastante alta.

GRÁFICO No 3. Balance de la Reserva Federal y el NFP desde enero 2003 hasta noviembre 2013.

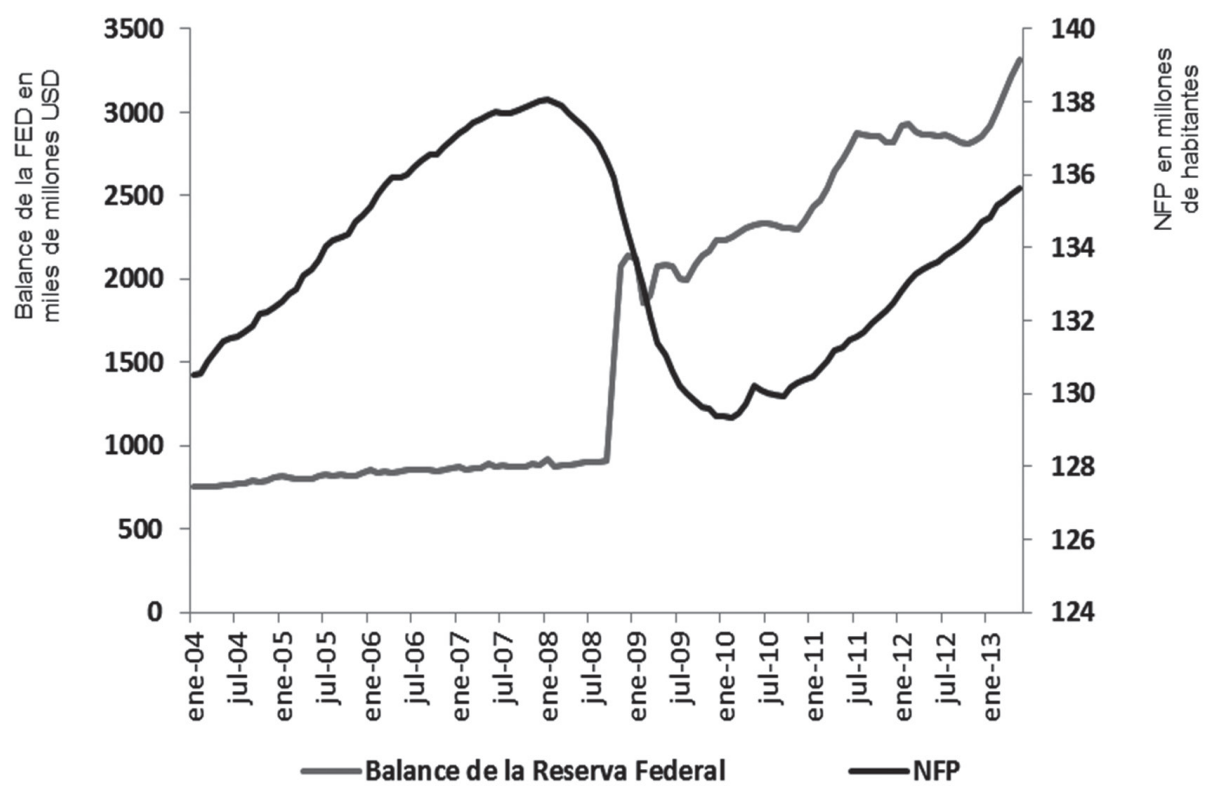

Fuente: Federal Reserve y Bureau of Economic Analysis Elaboración propia .

Las variaciones porcentuales del NFP, PBI y (B) -ver GRÁFICOS $\mathrm{N}^{\circ} 4^{\mathrm{a}}, 4 \mathrm{~b}$ y $4 \mathrm{c}$ - respectivamente, experimentan volatilidad dentro de bandas, manteniendo cierto estancamiento en su desempeño en el tiempo de estudio. 


\section{Carlos Palomino Selem}

CUADRO N ${ }^{\circ}$ a. Variación porcentual del NFP ( $\Delta \%$ NFP) entre 2003-Q1 y 2013-Q2.

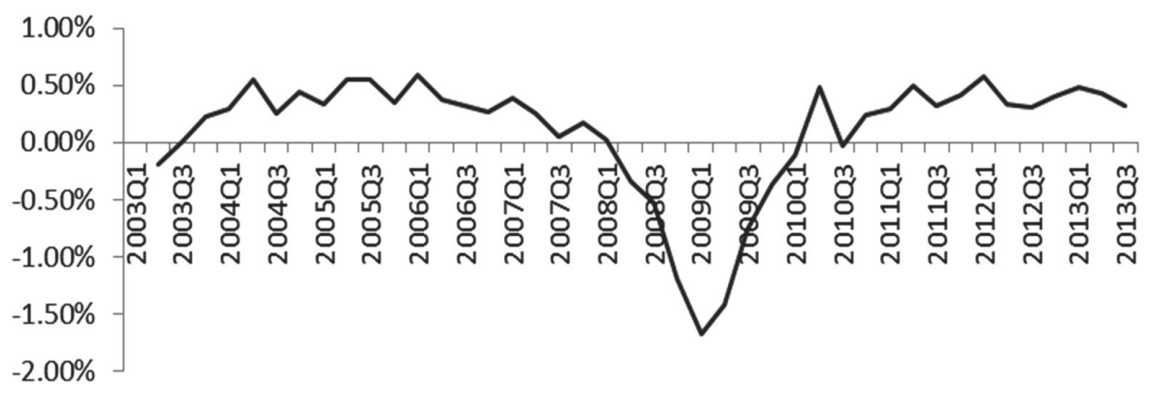

Fuente: Bureau of Economic Analysis.

Elaboración propia.

CUADRO N 4b. Variación porcentual del PBI ( $\Delta \%$ PBI) entre 2003-Q1 y 2013-Q2.

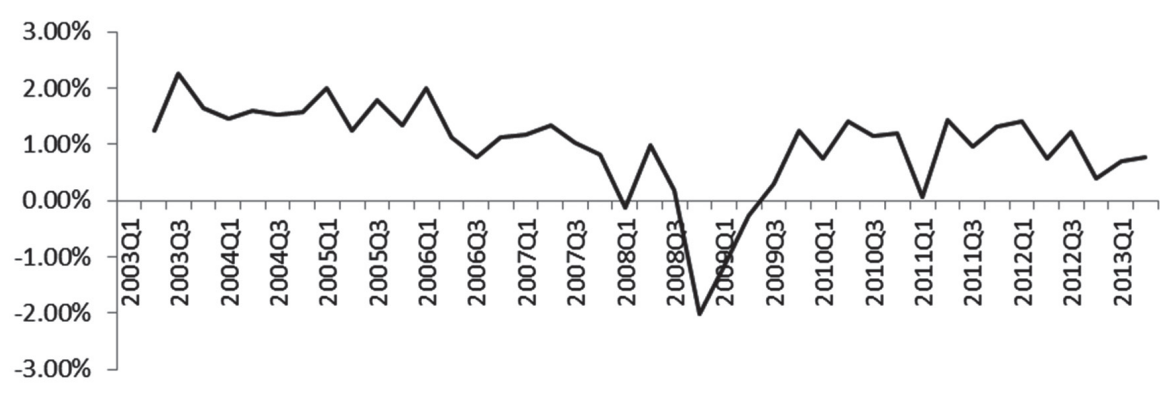

Fuente: Bureau of Economic Analysis

Elaboración propia. 


\section{Pensamiento Crítico Vol. 18. No 2}

CUADRO Nº 4c. Variación porcentual del Balance del Fed. ( $\Delta \% \mathrm{~B})$ entre 2003-Q1 y 2013-Q2.

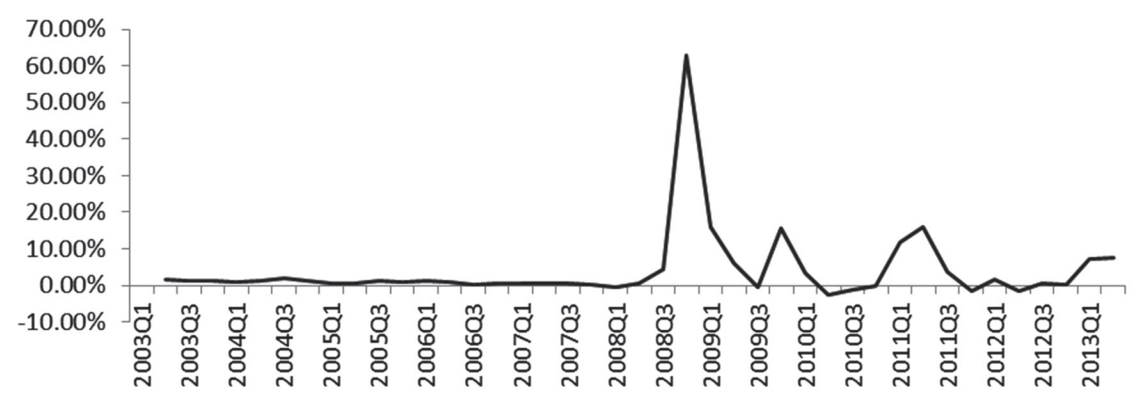

Fuente: Federal Reserve

Elaboración propia.

\section{LOS MODELOS DEL MARCO TEÓRICO}

\section{Modelo 1: NFP-B}

Sobre la base del marco teórico y la hipótesis propuesta hemos manejado un modelo basado en Mínimos Cuadrados Ordinarios (MCO) con rezagos. El modelo que mostramos en esta parte del estudio regresiona la variable de la NFP con el Balance del Fed. (B); como sigue:

$$
N F P_{t} / Q E=\beta_{0}+\beta_{1} * \beta_{\mathrm{t}-1}
$$

\footnotetext{
De la expresión (7), ${ }^{N F P_{t}} / Q E$ hace mención al carácter condicional de la estimación, el valor que toma el NFPt dado (/) QE, carácter de la política monetaria seguida para este periodo. Los resultados se encuentran en los CUADROS $N^{\circ} 1^{\mathrm{a}}, 1 \mathrm{~b}$ :
} 


\section{Carlos Palomino Selem}

CUADRO N 1 a. Relación entre el NFP y el Balance de la Fed. entre mayo del 2008 y mayo del 2005

\begin{tabular}{|c|c|c|c|c|}
\hline \multicolumn{5}{|c|}{$\begin{array}{l}\text { Dependent Variable: SERIES01 } \\
\text { Method: Least Squares } \\
\text { Date: } 11 / 22 / 13 \text { Time: } 14: 48 \\
\text { Sample: } 2008 \text { M01 2013M05 } \\
\text { Included observations: } 65 \\
\text { SERIES01 }=C(1)+C(2)^{*} \text { SERIES02-1 }\end{array}$} \\
\hline & Coefficient & Std. Error & t-Statistic & Prob. \\
\hline$C(1)$ & -543.6210 & 505.0143 & -1.076447 & 0.2858 \\
\hline$C(2)$ & 1.000738 & 0.000688 & 1454.532 & 0.0000 \\
\hline \multirow{7}{*}{$\begin{array}{l}\text { R-squared } \\
\text { Adjusted R-squared } \\
\text { S.E. of regression } \\
\text { Sum squared resid } \\
\text { Log likelihood } \\
\text { F-statistic } \\
\text { Prob(F-statistic) }\end{array}$} & 0.999970 & \multicolumn{2}{|c|}{ Mean dependent var } & 734014.7 \\
\hline & 0.999970 & \multicolumn{2}{|c|}{ S.D. dependent var } & 575.4630 \\
\hline & 3.165028 & \multicolumn{2}{|c|}{ Akaike info criterion } & 5.172487 \\
\hline & 631.0964 & \multicolumn{2}{|c|}{ Schwarz criterion } & 5.239391 \\
\hline & -166.1058 & \multicolumn{2}{|c|}{ Hannan-Quinn criter. } & 5.198885 \\
\hline & 2115664 . & \multirow{2}{*}{\multicolumn{2}{|c|}{ Durbin-Watson stat }} & 2.073941 \\
\hline & 0.000000 & & & \\
\hline
\end{tabular}

Fuente: Federal Reserve y US Bureau of Labor Statistic

Elaboración propia.

El término series hace referencia: series01 corresponde a la variable NFP y series 02 el (B).

La regresión (7) presenta resultados bastante aceptables en " $\mathrm{B}$ " y en los estadísticos R2, R2 -Ajustado y auto-correlación (Durbin-Watson,d); además, de altas correlación entre el NFP y Balance de la Fed. 


\section{Pensamiento Crítico Vol. 18. No 2}

CUADRO No 1b. Correlación entre el NFP y el Balance de la Fed. entre mayo del 2008 y mayo del 2005

\begin{tabular}{|c|c|c|}
\hline \multicolumn{3}{|c|}{ Correlación } \\
\hline & NFP & Balance Fed \\
\hline NFP & 1.000000 & 0.999985 \\
\hline Balance Fed & 0.999985 & 1.000000 \\
\hline
\end{tabular}

Elaboración propia.

Pero analizando la normalidad (ver GRÁFICO No 5 ) de los residuos esta es bastante baja; pero debe agregarse que el número de observaciones es pequeño, de 64, por debajo de 100, lo cual no necesariamente podría inferirse la no significancia del modelo. Respecto a la prueba F, estaría confirmando alternativamente cierta significancia del modelo.

GRÁFICO N ${ }^{\circ}$. Normalidad de los Residuos de las series del NFP y el Balance del Fed. entre el 2008-enero y 2013-mayo

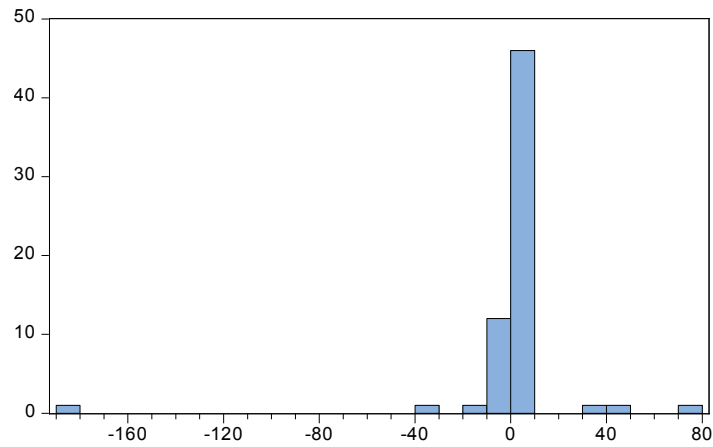

\begin{tabular}{lr} 
Series: RESID \\
Sample 2008M01 2013M05 \\
Observations 64 \\
Mean & $1.93 \mathrm{e}-15$ \\
Median & 1.483432 \\
Maximum & 72.93101 \\
Minimum & -189.0015 \\
Std. Dev. & 27.00172 \\
Skewness & -5.098721 \\
Kurtosis & 39.73954 \\
& \\
Jarque-Bera & 3876.751 \\
Probability & 0.000000 \\
\hline
\end{tabular}

Fuente: Federal Reserve y Bureau of Economic Analysis.

Elaboración propia.

\section{Modelo de Aceleración PBI-NFP-B}

Este se formula como sigue:

$$
\frac{d f^{\prime}(P B I)}{d t}=\beta_{0}+\beta_{1} \frac{d g^{\prime}(N F P)}{d t}+\beta_{2} \frac{d h^{\prime}(B)}{d t}
$$




\section{Carlos Palomino Selem}

Donde: $f(\mathrm{PBI})=\operatorname{Ln}(\mathrm{PBI}+\mathrm{k})$

$\mathrm{g}(\mathrm{NFP})=\operatorname{Ln}(\mathrm{NFP} t)$

$h(B)=\operatorname{Ln}(B t)$

donde "d" es el operador de derivada de la función respecto a "t" tiempo; y $\mathrm{K}=1$. La ecuación (8) expresa la relación entre la aceleración de B y la NFP con la aceleración del PBI la expresión (8) se puede, también, presentar como:

$Z=\beta_{0}+\beta_{1} Y+\beta_{2} X$

Los resultados encontrados se presentan en el cuadro $\mathrm{N}^{\circ} 2$.

CUADRO No 2. Relación de Aceleración del PBI, NFP y Balance del Fed. entre 2003-Q4 y 2013Q-2

Dependent Variable: VARGDP

Method: Least Squares

Date: 11/13/13 Time: 15:02

Sample (adjusted): 2003Q4 2013Q2

Included observations: 39 after adjustments

\begin{tabular}{lrlrr}
\hline \hline \multicolumn{1}{c}{ Variable } & Coefficient & Std. Error & t-Statistic & Prob. \\
\hline \hline \multicolumn{1}{c}{ C } & -0.618813 & 0.454658 & -1.361053 & 0.1820 \\
\multicolumn{1}{c}{ VARNFP_1 } & 0.042748 & 0.113667 & 0.376078 & 0.7091 \\
$\quad$ VARB_1 & -0.149878 & 0.023387 & -6.408507 & 0.0000 \\
\hline \hline R-squared & 0.533453 & Mean dependent var & -0.134475 \\
Adjusted R-squared & 0.507534 & S.D. dependent var & 3.963853 \\
S.E. of regression & 2.781670 & Akaike info criterion & 4.957783 \\
Sum squared resid & 278.5567 & Schwarz criterion & 5.085750 \\
Log likelihood & -93.67677 & Hannan-Quinn criter. & 5.003697 \\
F-statistic & 20.58135 & Durbin-Watson stat & 2.265965 \\
Prob(F-statistic) & 0.000001 & & \\
\hline \hline
\end{tabular}

$\mathrm{GDP}=\mathrm{PBI}$

Fuente: Federal Reserve, Bureau of Economic Analysis y US Bureau of Labor Statistic.

Elaboración propia. 


\section{Pensamiento Crítico Vol. 18. № 2}

Si bien el modelo presenta (ver cuadro $\mathrm{N}^{\circ} 2$ ) resultados estadísticamente buenos, el signo que toma el coeficiente de la aceleración de $\mathrm{B}$, esto descalifica al modelo teóricamente; dado que si bien $\frac{d h^{\prime}(B)}{d t}<0$, con el valor de $\beta_{2}<0$ puede decirse que la política monetaria se comportado menos expansiva en su ritmo debilitando la aceleración en el incremento del PBI, pero su efecto no fue contrario, reduciendo el crecimiento del PBI. Pero si $\frac{d h^{\prime}(B)}{d t}>0$, teóricamente se presentaría una inconsistencia.

Modelo Aceleración PBI-B

Establecido el modelo como sigue:

$\frac{d f^{\prime}(P B I)}{d t}=\beta_{0}^{A}+\beta_{1}^{B} \frac{d h^{\prime}(B)}{d t}$

Donde: $f(\mathrm{PBI})=\operatorname{Ln}(\mathrm{PBI}+\mathrm{k})$

$$
\mathrm{h}(\mathrm{B})=\operatorname{Ln}(\mathrm{Bt})
$$

La ecuación (10) puede, también, expresarse como sigue:

$$
Z_{1}=\beta_{0}^{A}+\beta_{1}^{B} X_{1}
$$

Al Igual que en el caso del modelo de aceleración PBI-NFP-B, el modelo de aceleración PBI-B presenta resultados estadísticamente buenos (ver cuadro $\mathrm{N}^{\circ} 3$ ), pero el signo que toma el coeficiente de la variable (B) es negativo. 


\section{Carlos Palomino Selem}

CUADRO N 3. Relación de Aceleración del PBI y Balance del Fed. entre 2003-Q4 y 2013Q-2

\begin{tabular}{|c|c|c|c|c|}
\hline \multicolumn{5}{|c|}{$\begin{array}{l}\text { Dependent Variable: VARGDP } \\
\text { Method: Least Squares } \\
\text { Date: } 11 / 13 / 13 \text { Time: } 14: 52 \\
\text { Sample (adjusted): } 2003 Q 42013 Q 2 \\
\text { Included observations: } 39 \text { after adjustments }\end{array}$} \\
\hline Variable & Coefficient & Std. Error & t-Statistic & Prob. \\
\hline C & -0.636339 & 0.446985 & -1.423623 & 0.1629 \\
\hline VARB_1 & -0.149782 & 0.023113 & -6.480414 & 0.0000 \\
\hline R-squared & 0.531620 & \multicolumn{2}{|c|}{ Mean dependent var } & -0.134475 \\
\hline Adjusted R-squared & 0.518962 & \multicolumn{2}{|c|}{ S.D. dependent var } & 3.963853 \\
\hline S.E. of regression & 2.749207 & \multicolumn{2}{|c|}{ Akaike info criterion } & 4.910422 \\
\hline Sum squared resid & 279.6511 & \multicolumn{2}{|c|}{ Schwarz criterion } & 4.995733 \\
\hline Log likelihood & -93.75323 & \multicolumn{2}{|c|}{ Hannan-Quinn criter. } & 4.941031 \\
\hline F-statistic & 41.99576 & \multicolumn{2}{|c|}{ Durbin-Watson stat } & 2.177113 \\
\hline Prob(F-statistic) & 0.000000 & & & \\
\hline
\end{tabular}

$\mathrm{GDP}=\mathrm{PBI}$

Fuente: Bureau of Economic Analysis y Federal Reserve

Elaboración propia.

\section{EVENTOS ESPECÍFICOS}

En esta parte se discutirá el motivo por el cual los modelo presentados en esta parte no presentan resultados coherentes o consistentes con la teoría.

Primero, parece que la política de estímulos monetarios no ha sido muy firmemente sostenida durante el proceso de recuperació ${ }^{\circ}$ En efecto, si observamos el GRÁFICO N ${ }^{\circ}$ 6 las variaciones importantes en B entre el 2008-Q3 y 2009-QE3 no tuvieron impacto en la misma magnitud sobre el NFP, si bien este mejoró, esto no varió en la misma proporción que $\mathrm{B}$, fue bastante menor, lo mismo sucede en los periodos comprendidos entre el 2010-Q4 al 2011-Q3 (ver circunferencias en el GRÁFICO) y en la coyuntura actual del 2013. 


\section{Pensamiento Crítico Vol. 18. № 2}

GRÁFICO No 6. Variaciones porcentual del NFP y B ( $4 \%$ NFP vs $\Delta \% B$ ) entre 2008-Q1 y 2013-Q2.

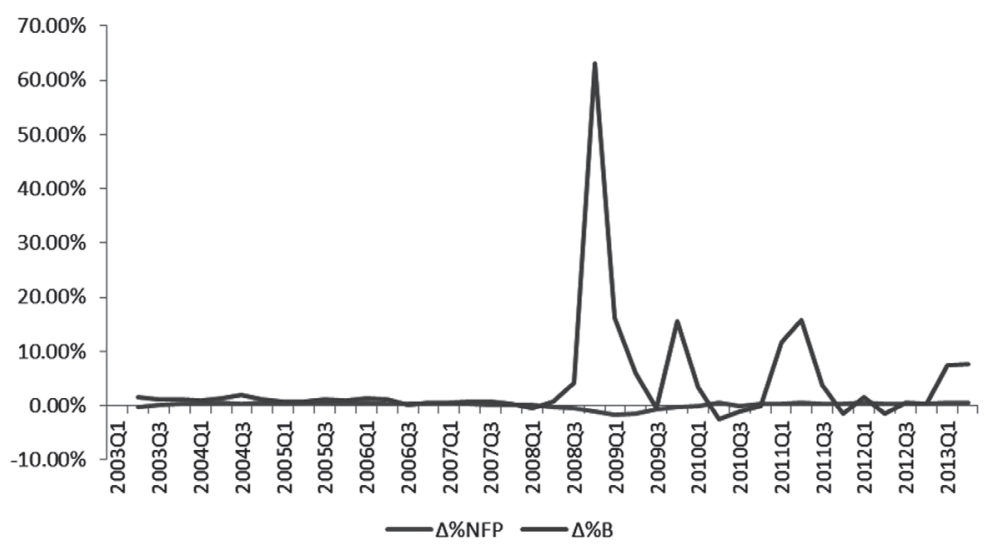

Fuente: US Bureau of Labor Statistic y Federal Reserve

Elaboración propia.

En el caso de la aceleración en B y su impacto en la aceleración del NFP, aquel no fue firme en los periodos de mejora que experimentó el NFP (ver GRÁFICO N 7a, circunferencias y $7 b)$.

GRÁFICO No 7a. Aceleración del NFP y B ( $\Delta \Delta \%$ NFP vs $\Delta \Delta \%$ B) entre 2008-Q1 y 2013-Q2.

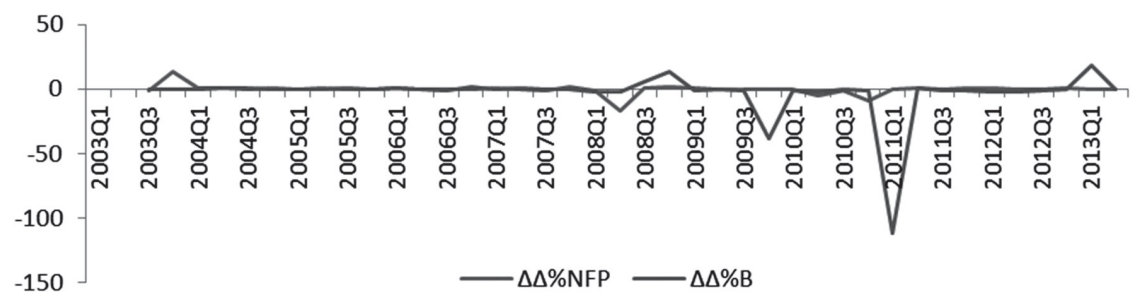

Fuente: US Bureau of Labor Statistic

Elaboración propia. 


\section{Carlos Palomino Selem}

Mirando el periodo 2008-Q1 hasta el 2008-Q3, se observa un retardo en reconocer el problema del empleo por parte del Fed. que desde el 2007-Q2 ya se conocía del problema financiero de los bancos; igual poca consistencia entre el periodo 2010-Q3 y 2011-Q1.

GRÁFICO $\mathrm{N}^{\circ} 7 \mathrm{~b}$. Aceleración del NFP y B ( $\Delta \Delta \% \mathrm{NFP}$ vs $\Delta \Delta \% \mathrm{~B}$ ) considerando los periodos de estímulos monetarios.

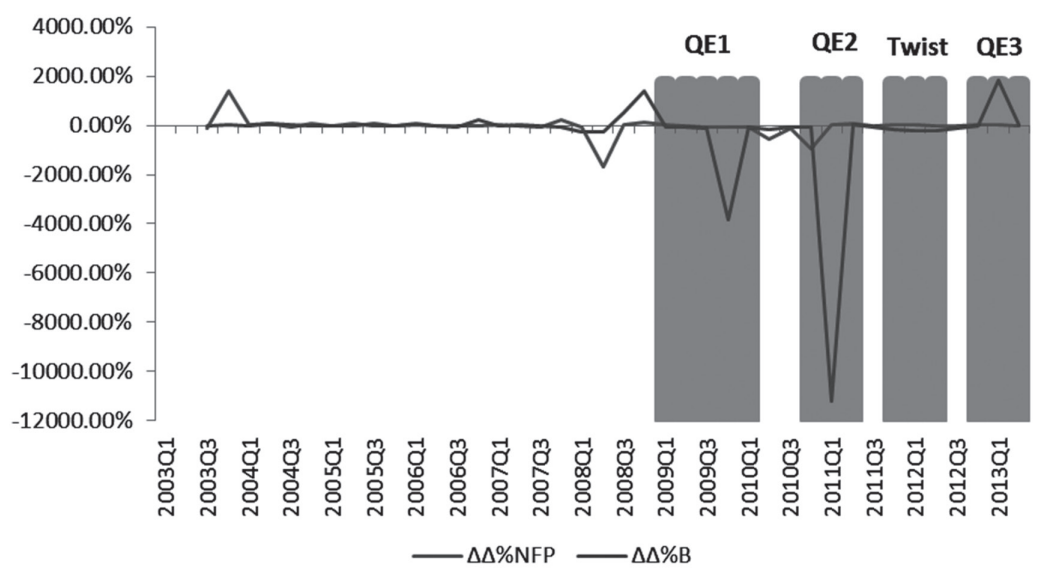

Fuente: US Bureau of Labor Statistic y Federal Reserve.

Elaboración propia.

\section{CONCLUSIONES Y RECOMENDACIONES}

Amanera de conclusiones se puede afirmar que existe una relación entre el NFP y el Balance del Fed, pero esta no parece ser de significancia para el periodo de estudio, en términos estadísticos no presenta normalidad en la distribución de sus residuos. La política monetaria de flexibilización cuantitativa, no parece haberse implementado de forma firme y consistente en el periodo de crisis y recuperación, lo cual confirmaría los resultados econométricos obtenidos por la prueba de normalidad.

Ninguno de los modelos planteados en la presente investigación alcanzó significancia estadística, pero más que por el tamaño de la data muestral, parece deberse 


\section{Pensamiento Crítico Vol. 18. No 2}

a problemas de índole práctico; es decir, no se observa continuidad y consistencia entre la política monetaria, en su diseño e implementación, y el empleo.

\section{REFERENCIAS BIBLIOGRÁFICAS}

FOMC Meetings (2013). Varios Statemente sobre las Minutes, en:

http://www.federalreserve.gov/monetarypolicy/fomccalendars.htm.

Marc Chandler (2013). Non Farm Payrolls Mix Fed, en:

http://www.cmegroup.com/education/market-commentary/fx/2013/10/mid-sessionfx_16257.html

Yelft Cox (2013). Shutdown slowdown? Job creation soars in October; en: http://www.cnbc.com/id/101182289 .

Bureau of Economic Analysis (2013). Estadística del PIB EEUU. Varios años: http://www.bea.gov/iTable/iTable.cfm?ReqID=9\&step=1\#reqid=9\&step=3\&isuri $=$ $1 \& 910=X \& 911=0 \& 903=5 \& 904=1992 \& 905=2013 \& 906=Q$.

Federal Reserve (2013). Estadística Base Monetaria. Varios años:

http://www.federalreserve.gov/datadownload/Build.aspx?rel=H3.

Federal Reserve (2013). Estadística Balance del Fed. Varios años:

http://www.federalreserve.gov/datadownload/Download.aspx?rel $=H 41 \&$ series $=\mathrm{a} 0$ ec2eb5aa72c1a6cd989904eb57812a\&filetype $=$ csv\&label $=$ include\&layout $=$ series column\&from $=01 / 01 / 2003 \&$ to $=11 / 22 / 2013$.

US Bureau of Labor Statistic (2013). Estadísitica Non Farm Payroll. Varios años: http://www.bls.gov/webapps/legacy/cesbtab1.htm. 